\title{
Two Chaotic Patterns of Dynamic Risk Definition for Solving Hazardous Materials Routing Problem
}

\author{
Abbas Mahmoudabadi \\ Department of Industrial Engineering, Mehrastan University, Kisom Road, Astaneh-ye Ashrafiyeh, Gilan, Iran \\ Correspondence should be addressed to Abbas Mahmoudabadi; mahmoudabadi@phd.pnu.ac.ir
}

Received 7 May 2014; Revised 2 November 2014; Accepted 16 December 2014

Academic Editor: Lihong Zhang

Copyright ( 2015 Abbas Mahmoudabadi. This is an open access article distributed under the Creative Commons Attribution License, which permits unrestricted use, distribution, and reproduction in any medium, provided the original work is properly cited.

In the case of determining routes for hazardous material transportation, risk is considered as a main attribute. Transport risk, which is usually combined with other attributes such as cost or travel time, plays a significant role in determining paths for hazardous materials transportation. Since, risk is chaotically affected by road incidents, decision makers are dealing with selecting a method for defining chaotic risk factors in hazmat transportation. In this paper, transport risk has been defined as a chaotic variable using two different methods of generating chaotic patterns. In an experimental road network, which consists of eighty-nine nodes and one hundred and one two-way links, two different methods of generating chaotic variables have been used for applying the proposed procedure. In addition, results for different amounts of risk and cost have also been analyzed in case study. Results revealed that different cost and risk priorities change the frequencies of selected paths determined for hazmat transportation, but the route convergence of the route to chaos method is better than that of the logistic map equation.

\section{Introduction}

1.1. Hazmat Routing Problem. Hazardous materials (hazmat for short) which are classified into nine classes of industrial and natural substances [1] are involved in a large part of the economic activities, not only in industrialized [2] but also in developing countries [3]. Management of hazardous material is usually considered as an extremely complex issue involving a multitude of environmental, engineering, economic, social, and political concerns [4] due to their incident impacts. One of the most important issues in hazardous material management is to find the best path for transportation which is known as hazmat routing problem (HRP). HRP is usually a double-sided consideration problem in which local authorities are interested in minimizing public risk and carriers are dealing with minimizing transport cost [5]. In this case, a combination of risk and cost is usually used for developing mathematical models in order to find the best path for hazmat transportation. Mathematical modeling result in this matter is not necessarily the shortest path. Relevant studies show that different combinations of risk and cost lead researchers to use various methods for solving HRP $[6,7]$ and different priorities for risk and cost or travel time change results on the above-mentioned problem [3].

1.2. Chaos Theory. Edvard Lorenz introduced the concept of chaos theory in 1963. He found the chaotic attractions in complex system of weather forecasting when he entered different values as starting points in computer program [8]. Chaos has also been studied within the engineering scientific and mathematical communities and was found to be useful in many disciplines such as high-performance circuits and devices, collapse prevention of power systems, and also information processing [9]. While chaos theory is used to analyze complex systems and transportation systems are complex entities [10], it may be found useful for applying in the field of transportation. In transportation systems, considering legal and social constraints helps researchers more accurately predict human actions and system evolution $[11,12]$.

Following the utilization of chaos theory in transportation and road safety, it has been approved that the behavior of road incident over the road network is chaotic $[3,13]$ and showed that the ratio of the fatal accidents in a specific part of road network to all accidents has a chaotic behavior which 
could be defined as a chaotic variable. The normal form of logistic map equation is used to generate dynamic risk factors over a year and applied the concept of chaos theory to define dynamic risk factors $[3,13]$ in which the Lyapunov characteristic exponent was calculated for checking the presence of chaos $[13,14]$.

There are some equations used for generating chaotic variables in complex systems. Logistic map [9] and route to chaos [15] equations are well known. Logistic map equation is formulated by the following equation, where $R(t)$ is the chaotic variable at time $t$ and $K_{l}$ is chaos parameter mainly set to 4 [9]:

$$
\begin{array}{r}
R(t)=K_{l} \times R(t-1) \times(1-R(t-1)) \\
\forall t \in \text { Defined Time Interval. }
\end{array}
$$

Route to chaos equation is formulated by the following equation, where $R(t)$ is chaotic variable at time $t$ and $K_{r}$ is also defined as chaos parameter mainly set to approximate interval 1.0624-1.0807 [15]:

$$
\begin{array}{r}
R(t)=K_{r} \times R(t-1)^{2}+0.3 \times R(t-2) \\
\forall t \in \text { Defined Time Interval. }
\end{array}
$$

Considering different durations is the most important difference between the above equations. Logistic map equation uses only the previous time while route to chaos equation is dependent on the previous and the preprevious terms of chaotic variable.

1.3. Vision. Following the above sections, it is concluded that transport risk is an important attribute in hazardous material transportation planning [16]. On the other hand, recent studies show that the risk regarding the road accidents can be defined as a chaotic variable in terms of probability and hazmat incident impacts [3]. Further research also shows that chaotic behavior of risk can be used for defining risk when a part of road network has been affected by natural disasters such as flood, heavy rain, or earthquake [17]. Risk factors in the previous studies are mainly formulated using logistic map equation, so utilizing the other structures of chaotic variables is an attractive topic in this field. Therefore, the aim of this research work is to apply both logistic map and route to chaos equations for defining chaotic risk variables in HRP. As a main criterion, path convergence according to the most frequent path is considered in order to check chaotic generation performances of the above equations.

\section{Defining Procedure}

Because of the existing chaotic behavior in daily road accidents [13], risk factors, associated with the edges (links) of network, are different over a year [3] and different paths will be outlined for hazmat transportation, accordingly. Therefore, the proposed procedure is capable of considering different chaotic variables over the network. In order to cover a wide range of variations, the proposed procedure

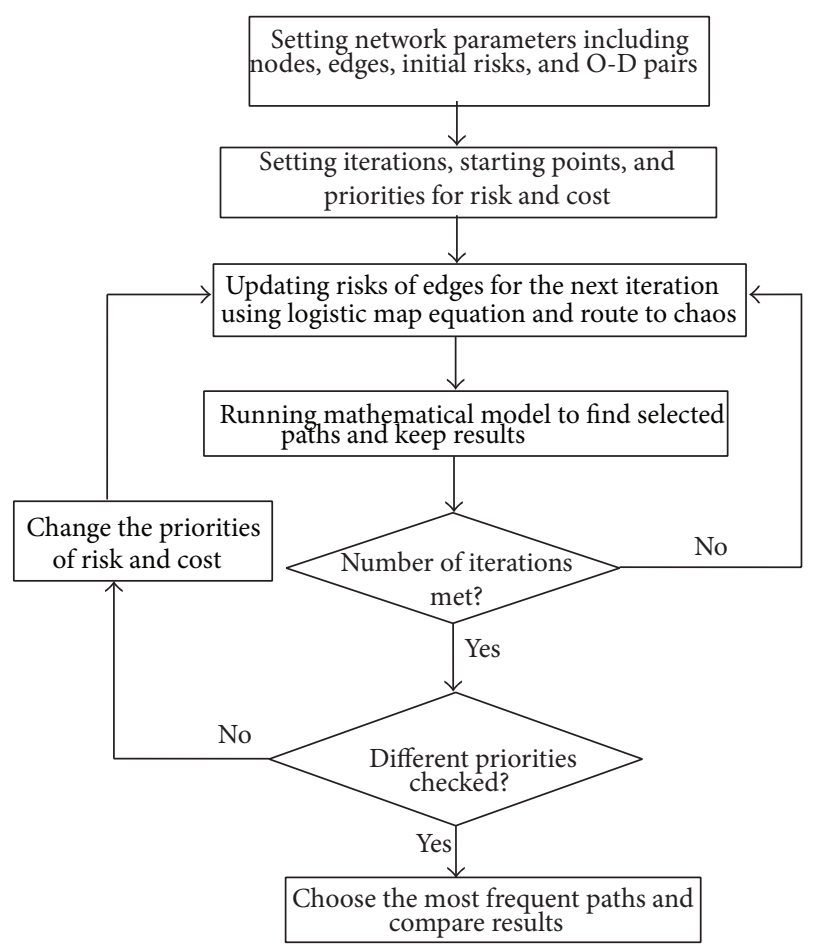

FIGURE 1: Overall view of the proposed procedure.

is designed as an iterative based method for solving HRP in which a wide range of risk and cost priorities are simultaneously used to find the best path over a year. The total combination of risk and cost priorities is defined as criterion in HRP objective function. Risk factors are updated utilizing logistic map and route to chaos equations over a specific number of iterations (such as 365 days over a year). Different priorities of risk and cost are also considered in the proposed procedure. Termination is decided by both iteration number and combinations of risk and cost. In other words, the proposed procedure is terminated when the number of iterations is met for all combinations of risk and cost. The most frequent selected path is eventually outlined to be considered as the best path for HRP in terms of their repetitions. Figure 1 shows an overall view of the proposed procedure including six steps as follows.

(1) Set network parameters including nodes, edges (links), uniformed risk, and cost for each edge.

(2) Set model parameters including number of iterations, starting points, and priorities for risk and cost.

(3) Update dynamic risk factors for all edges to the next iteration (day) using logistic map and route to chaos equations.

(4) Run mathematical model to find the best path for different priorities of risk and cost and keep them according to dynamic risks outlined by both generation equations. 
(5) If stopping criteria (the number of iterations and different priorities of risk and cost) are met, stop and go to Step 6; otherwise go back to Step 3.

(6) Choose the most frequent paths and compare the results.

\section{Developing Mathematical Model}

Assume that the road network is defined by graph $G(n, e)$ including predefined nodes $(n)$ and edges $(e)$. Nodes are categorized in to three main sets including origin, destination, and connecting nodes. Following the concept defined in Section 2, mathematical model is formulated to minimize the total combinations of risk and cost over the network. The uniform amounts of risk and cost should be used because of the existing different scales of variables in the proposed mathematical model, so the following equation is used to convert data into closed interval [ $\left.\begin{array}{ll}0.05 & 0.95\end{array}\right]$ :

$$
X_{\text {new }}=\frac{\left(X_{\text {old }}-X_{\text {min }}\right)}{\left(X_{\text {max }}-X_{\text {min }}\right)} * 0.9+0.05 \text {, }
$$

where $X_{\text {new }}$ is the uniformed amount of variable $X_{\text {old }}$, while $X_{\max }$ and $X_{\min }$ are the maximum and minimum of variable $X$, respectively [3].

In formulation process, $X_{i j}(t)$ is defined as a binary variable. If $X_{i j}(t)$ is assigned by 1 , link $(i, j)$ is located in the selected path; otherwise it is assigned by 0 . There are two attributes of cost and risk for each link defined as $C_{i j}$ and $R_{i j}$, respectively. As risk is updated in each iteration, it corresponds to $t$ notated by $R_{i j}(t) . P_{c}$ and $P_{r}$ are also, respectively, defined to cost and risk priorities. Following the above notations, objective function which defines the total amount combinations of cost and risk is formulated as (4).

The best path is the most frequent path, in which

$$
\begin{aligned}
& \operatorname{Min} Z=\sum_{G}\left[P_{r} \times R_{i j}(t)+P_{c} \times C_{i j}\right] \times X_{i j}(t) \\
& \forall(i, j) \in G, \quad t=1,2, \ldots, \text { Number of iterations, }
\end{aligned}
$$

where $t$ is iteration number.

There are some constraints in developing mathematical model. The main constraint is to keep continuous paths over the network. The concept can be formulated as [18]

$$
\begin{aligned}
\sum_{G} X_{i j}(t)-\sum_{G} X_{j i}(t)= \begin{cases}1 & \text { if } j \text { is an origin node } \\
-1 & \text { if } j \text { is a destination node } \\
0 & \text { otherwise }\end{cases} \\
\forall j \in G, t=1,2, \ldots, \text { Number of iterations. }
\end{aligned}
$$

The other equations are regarding chaotic behavior of risk associated with the network links. At the first round, onedimensional logistic map equation is used for updating risk in analytical process formulated by (6). In the second round, route to chaos equation is also used for generating chaotic variables using (7). Both parameters $K_{l}$ and $K_{r}$ are defined as chaotic variables for the following equations:

$$
\begin{array}{r}
R_{i j}(t)=K_{l} \times R_{i j}(t-1) \times\left(1-R_{i j}(t-1)\right) \\
\forall t=1,2, \ldots, \text { Number of iterations, } \\
R_{i j}(t)=K_{r} \times R_{i j}(t-1)^{2}+0.3 \times R_{i j}(t-2) \\
\forall t=1,2, \ldots, \text { Number of iterations. }
\end{array}
$$

Eventually, in intercity transportation, two-way edges are usually available, so the following equation is inserted in mathematical model:

$$
(i, j),(j, i) \in G
$$

To summarize the above notations and descriptions, mathematical model is formulated as follows:

$$
\begin{aligned}
& \text { Min } \quad Z=\sum_{G}\left[P_{r} \times R_{i j}(t)+P_{c} \times C_{i j}\right] \times X_{i j}(t) \\
& \forall(i, j) \in G, \quad t=1,2, \ldots, \text { Number of iterations, } \\
& \text { S.T. } \quad \sum_{G} X_{i j}(t)-\sum_{G} X_{j i}(t)= \begin{cases}1 & \text { if } j \text { is an origin node } \\
-1 & \text { if } j \text { is a destination node } \\
0 & \text { otherwise }\end{cases} \\
& R_{i j}(t)=K_{l} \times R_{i j}(t-1) \times\left(1-R_{i j}(t-1)\right) \\
& \forall t=1,2, \ldots, \text { Number of iterations, } \\
& R_{i j}(t)=K_{r} \times R_{i j}(t-1)^{2}+0.3 \times R_{i j}(t-2) \\
& \forall t=1,2, \ldots, \text { Number of iterations, } \\
& (i, j),(j, i) \in G, \quad X_{i j} \text { Binary variable. }
\end{aligned}
$$

\section{Case Study and Experimental Data}

Iranian north-west part including six provinces of West Azarbayegan, East Azarbayegan, Ardabil, Kordestan, Zanjan, and Hamadan has been selected as case study. Selected network includes 89 nodes and 101 two-way links as shown in Figure 2. Some nodes are border nodes which connect research area to other provinces. Vehicles will be assigned to transport hazardous materials subject to minimizing the combination of risk and cost in which different amounts of priorities are used over the network. More details on risk components and their assessment method are available in [3].

The other issue which is regarding the problem definition is to define origin-destination pairs. Two sets of origindestination pairs have been used to check the proposed procedure and develop mathematical model. The first set includes origin node 43 (Orumieh) and destination node 71 (Khodabandeh) and the second consists of origin node 33 (Ardabil) and destination node 67 (Sanandaj). 
TABLE 1: Best and secondary optimal paths and lengths from Orumieh (node 43) to Khodabandeh (node 71).

\begin{tabular}{|c|c|c|c|c|c|}
\hline Chaos equation & $(\text { Cost, risk) })^{*}$ & Solution ranking & Ranked most probable path & Length (KM) & $F^{* *}$ \\
\hline \multirow{11}{*}{ Logistic } & \multirow{2}{*}{$(0,1)$} & Best optimal & 438454535960657275897471 & 644 & 262 \\
\hline & & Secondary optimal & 4384545358616485657275897471 & 630 & 27 \\
\hline & \multirow{2}{*}{$(0.3,0.7)$} & Best optimal & 438454535960657275897471 & 644 & 288 \\
\hline & & Secondary optimal & 4384545358616485657275897471 & 630 & 29 \\
\hline & \multirow{2}{*}{$(0.5,0.5)$} & Best optimal & 438454535960657275897471 & 644 & 302 \\
\hline & & Secondary optimal & 4384545358616485657275897471 & 630 & 33 \\
\hline & \multirow{2}{*}{$(0.7,0.3)$} & Best optimal & 438454535960657275897471 & 644 & 319 \\
\hline & & Secondary optimal & 4384545358616485657275897471 & 630 & 41 \\
\hline & \multirow{2}{*}{$(1,0)$} & Best optimal & 438454535960657275897471 & 644 & 365 \\
\hline & & Secondary optimal & No path & & \\
\hline & Best solution & & $(438454535960657275897471)$ & 644 & \\
\hline \multirow{11}{*}{ Route to chaos } & \multirow{2}{*}{$(0,1)$} & Best optimal & 438454535960657275897471 & 644 & 301 \\
\hline & & Secondary optimal & 4384545358616485657275897471 & 630 & 58 \\
\hline & \multirow{2}{*}{$(0.3,0.7)$} & Best optimal & 438454535960657275897471 & 644 & 304 \\
\hline & & Secondary optimal & 4384545358616485657275897471 & 630 & 44 \\
\hline & \multirow{2}{*}{$(0.5,0.5)$} & Best optimal & 438454535960657275897471 & 644 & 305 \\
\hline & & Secondary optimal & 4384545358616485657275897471 & 630 & 51 \\
\hline & \multirow{2}{*}{$(0.7,0.3)$} & Best optimal & 438454535960657275897471 & 644 & 336 \\
\hline & & Secondary optimal & 4384545358616485657275897471 & 630 & 12 \\
\hline & \multirow{2}{*}{$(1,0)$} & Best optimal & 438454535960657275897471 & 644 & 365 \\
\hline & & Secondary optimal & No path & & \\
\hline & Best solution & & $(438454535960657275897471)$ & 644 & \\
\hline
\end{tabular}

${ }^{*}$ (Cost, risk) priority set is ordered by left to right.

${ }^{* * *}$ Frequency is regarding 365 iterations.

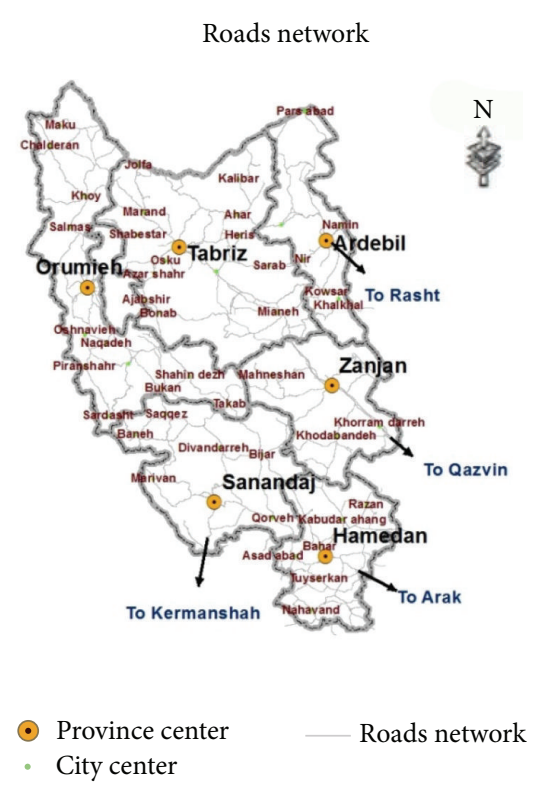

FIGURE 2: An overall geographical map of case study.

\section{Running Model and Discussion}

Mathematical model which has been exactly explained and developed in Section 3 can be solved using the well-known software of General Algebraic Modeling System (GAMS). The above-mentioned software enables authors to use the "loop command" in mathematical models and recalculate chaotic risk factors iteratively. Since two sets of origin-destination pairs have been selected for running the model, results are tabulated in Tables 1 and 2. Two different equations of logistic map and route to chaos have been utilized for each origindestination pair. In this way, logistic map and route to chaos equations have been applied for generating chaotic variables of risk in the first and second running times, respectively.

Results include two types of selected paths. Ranking selected paths according to their frequencies makes the most frequent path be known as the best optimal solution and next path be known as the secondary optimal solution. In other words, the best optimal solution shows a path with the most frequent selection using procedure and the secondary optimal solution follows in terms of the number of frequency. Both optimal and secondary optimal solutions are shown in Tables 1 and 2.

As observed, Tables 1 and 2 consist of the best and the secondary optimal solutions represent selected paths according to their frequencies. Frequencies are also available in the last column of each table extracted from 365 iteration solutions. Different priorities for cost and risk are also considered in the second column to cover a wide range. The number of iterations is set to 365 to satisfy decision makers in order to cover various amounts of risk factors over a year.

According to what discussed in Section 3, considering two different attributes makes a multiobjective function in 
TABLE 2: Best and secondary optimal paths and their lengths from Ardabil (node 33) to Sanandaj (node 67).

\begin{tabular}{|c|c|c|c|c|c|}
\hline Chaos equation & $\left(\right.$ Cost, risk) ${ }^{*}$ & Solution ranking & Ranked most probable path & Length (KM) & $F^{* *}$ \\
\hline \multirow{11}{*}{ Logistic map } & \multirow{2}{*}{$(0,1)$} & Best optimal & 333437383940424445505152535960658567 & 783 & 74 \\
\hline & & Secondary optimal & 333437383940424445505152535861648567 & 657 & 63 \\
\hline & \multirow{2}{*}{$(0.3,0.7)$} & Best optimal & 333437383940424445505152535861648567 & 657 & 116 \\
\hline & & Secondary optimal & 333437383940424445505152535960658567 & 783 & 97 \\
\hline & \multirow{2}{*}{$(0.5,0.5)$} & Best optimal & 333437383940424445505152535861648567 & 657 & 185 \\
\hline & & Secondary optimal & 333437383940424445505152535960658567 & 783 & 93 \\
\hline & \multirow{2}{*}{$(0.7,0.3)$} & Best optimal & 333437383940424445505152535861648567 & 657 & 318 \\
\hline & & Secondary optimal & 333437383940424445505152535960658567 & 783 & 35 \\
\hline & \multirow{2}{*}{$(1,0)$} & Best optimal & 333437383940424445505152535861648567 & 657 & 365 \\
\hline & & Secondary optimal & No path & & \\
\hline & Best solution & & (33 3437383940424445505152535861648567$)$ & 644 & \\
\hline \multirow{11}{*}{ Route to chaos } & \multirow{2}{*}{$(0,1)$} & Best optimal & 3334373839404244455046834782487572658567 & 1023 & 99 \\
\hline & & Secondary optimal & 333437383940424445505152535960658567 & 783 & 83 \\
\hline & \multirow{2}{*}{$(0.3,0.7)$} & Best optimal & 333437383940424445505152535960658567 & 783 & 162 \\
\hline & & Secondary optimal & 3334373839404244455046834782487572658567 & 1023 & 60 \\
\hline & \multirow{2}{*}{$(0.5,0.5)$} & Best optimal & 333437383940424445505152535960658567 & 783 & 207 \\
\hline & & Secondary optimal & 3334373839404244455046834782487572658567 & 1023 & 78 \\
\hline & \multirow{2}{*}{$(0.7,0.3)$} & Best optimal & 333437383940424445505152535960658567 & 783 & 348 \\
\hline & & Secondary optimal & 3334373839404244455046834782487572658567 & 1023 & 11 \\
\hline & \multirow{2}{*}{$(1,0)$} & Best optimal & 333437383940424445505152535960658567 & 783 & 365 \\
\hline & & Secondary optimal & No path & & \\
\hline & Best solution & & (33 3437383940424445505152535960658567$)$ & 783 & \\
\hline
\end{tabular}

${ }^{*}$ (Cost, risk) priority set is ordered by left to right.

${ }^{* *}$ Frequency is regarding 365 iterations.

TABLE 3: Frequency of the best optimal paths for different chaos equations.

\begin{tabular}{lcccccc}
\hline \multirow{2}{*}{ Chaos equation } & O-D & \multicolumn{5}{c}{ Cost, risk) priorities } \\
& Pairs & $(0,1)$ & $(0.3,0.7)$ & $(0.5,0.5)$ & $(0.7,0.3)$ & $(1,0)$ \\
\hline \multirow{2}{*}{ Logistic map } & $43-71$ & 262 & 288 & 302 & 319 & 365 \\
& $33-67$ & 74 & 116 & 185 & 318 & 365 \\
\hline \multirow{2}{*}{ Route to chaos } & $43-71$ & 301 & 304 & 305 & 336 & 365 \\
& $33-67$ & 99 & 162 & 207 & 348 & 365 \\
\hline \multirow{2}{*}{$\begin{array}{l}\text { Logistic map } \\
\text { Route to chaos }\end{array}$} & Average & 168 & 202 & 243.5 & 318.5 & 365 \\
& & 200 & 233 & 256 & 342 & 365 \\
\hline
\end{tabular}

mathematical model. Although there are some methods for solving multiobjective programs (such as preemptive goal programming, weighted objective function), uniform amounts of variables have been used for developing mathematical model in this research work.

For more discussion, results which are tabulated in Tables 1 and 2 are summarized in Table 3 and graphically shown in Figure 3. The average frequency for each O-D pair is selected for discussion. As it can be observed, average frequency is raised when priority of risk is decreased. It goes up to 365 when risk has no effect to find hazmat transport path. The other finding is corresponding to the path convergence derived by the proposed procedure. Path convergence criterion for using route to chaos equation is much better than the one for logistic map equation. The main

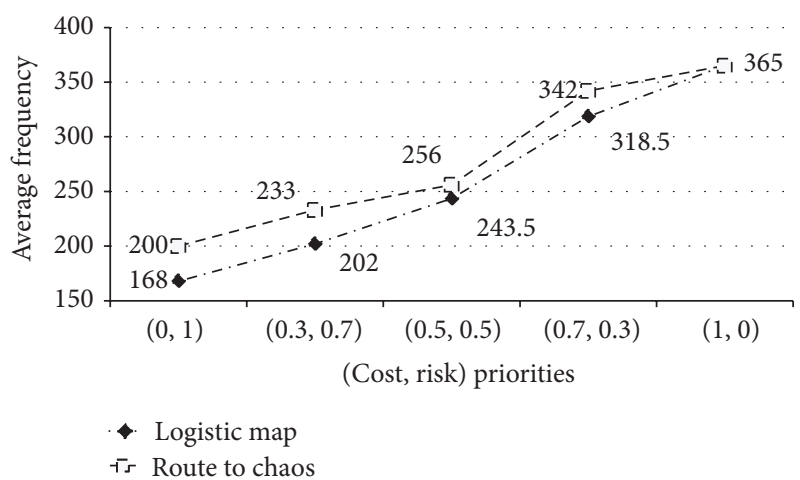

FIGURE 3: Average path frequency using methods of generating chaotic patterns.

reason is that the chaotic variable in logistic map equation is just dependent on the previous time but it is dependent on the previous and preprevious times in route to chaos equation. Therefore, using route to chaos equation for considering dynamic risk factors in hazardous material transport planning is recommended to researchers who work in this field.

\section{Conclusion}

In this research work, an iterative procedure, proposed following the basic principle of hazmat routing problem and 
the concept of chaos theory in dynamic risk definition, has been proposed and applied in hazmat transportation routing problem. Transport cost and risk have been considered as main attributes in HRP, so mathematical model has been developed to be capable of considering both risk and cost attributes. Risk is defined as a chaotic variable using two methods of generating chaotic variables including logistic map and route to chaos equations.

An experimental data set in a real road network, including eighty-nine nodes and one hundred and one two-way edges, has been used as a case study for analytical and evaluation process. The proposed procedure and mathematical model have been run in prespecified iteration number and origin-destination pairs. Results revealed that there are some differences between results when different equations for generating chaotic factors are used. It is also concluded that route to chaos equation is capable of determining the best path in better convergence rather than logistic map equation, because route to chaos equation uses two previous situations of chaotic variable but logistic map uses only the consecutive previous situation.

Further study may focus on the process of generating risk factors using different equations mainly utilized in the field of chaos theory. In a large network, since running time could be a concern, future research is also recommended to check the speed of procedure with more iterations and improve that for shorter running time.

\section{Conflict of Interests}

No funding or financial support from fellowship, scholarship, or any sponsors have been gained by the author. Therefore, the author declares that there is no conflict of interests regarding the publication of this research work.

\section{References}

[1] Environmental Health \& Safety, Hazardous Material Classification, NC State University, 2011, http://www.ncsu.edu/ehs/dot/ classification.html.

[2] K. G. Zografos and K. N. Androutsopoulos, "A heuristic algorithm for solving hazardous materials distribution problems," European Journal of Operational Research, vol. 152, no. 2, pp. 507-519, 2004.

[3] A. Mahmoudabadi and S. M. Seyedhosseini, "Developing a chaotic pattern of dynamic Hazmat routing problem," IATSS Research, vol. 37, no. 2, pp. 110-118, 2014.

[4] J. M. Díaz-Báñez, F. Gómez, and G. T. Toussaint, "Computing shortest paths for transportation of hazardous materials in continuous spaces," Journal of Food Engineering, vol. 70, no. 3, pp. 293-298, 2005.

[5] E. Erkut and O. Alp, "Designing a road network for hazardous materials shipments," Computers and Operations Research, vol. 34, no. 5, pp. 1389-1405, 2007.

[6] S. Bonvicini and G. Spadoni, "A hazmat multi-commodity routing model satisfying risk criteria: a case study," Journal of Loss Prevention in the Process Industries, vol. 21, no. 4, pp. 345358, 2008.

[7] A. S. Mohaymany and M. Khodadadian, "A routing methodology for hazardous material transportation to reduce the risk of road network," IUST International Journal of Engineering Science, vol. 19, no. 3, pp. 57-65, 2008.

[8] G. James, Chaos: Making a New Science, Vintage Publishing, 1987.

[9] J. Mingjun and T. Huanwen, "Application of chaos in simulated annealing," Chaos, Solitons \& Fractals, vol. 21, no. 4, pp. 933-941, 2004.

[10] W. L. Lawrence, Y. L. Feng, and Y. C. Huang, "Diagnosis of freeway traffic incidents with chaos theory," Journal of the Eastern Asia Society for Transportation Studies, vol. 5, pp. 20252038, 2003.

[11] C. Frazier and K. M. Kockelman, "Chaos theory and transportation systems: instructive example," Journal of the Transportation Research Board, no. 1897, pp. 9-17, 2004.

[12] G. Sugihara and R. M. May, "Nonlinear forecasting as a way of distinguishing chaos from measurement error in time series," Nature, vol. 344, no. 6268, pp. 734-741, 1990.

[13] A. Mahmoudabadi and S. M. Seyedhosseini, "Application of chaos theory in hazardous material transportation," International Journal of Transportation Engineering, vol. 1, no. 1, pp. 1523, 2012.

[14] L. D. Kiel and E. Elliott, Chaos Theory in the Social Sciences, Foundations and Applications, University of Michigan, 1996.

[15] C. Grebogi, E. Ott, and J. A. Yorke, "Crises, sudden changes in chaotic attractors, and transient chaos," Physica D: Nonlinear Phenomena, vol. 7, no. 1"3, pp. 181-200, 1983.

[16] E. Erkut and A. Ingolfsson, "Transport risk models for hazardous materials: revisited," Operations Research Letters, vol. 33, no. 1, pp. 81-89, 2005.

[17] A. Mahmoudabadi and S. M. Seyedhosseini, "Solving Hazmat Routing Problem in chaotic damage severity network under emergency environment," Transport Policy, vol. 36, pp. 34-45, 2014.

[18] E. Erkut and F. Gzara, "Solving the hazmat transport network design problem," Computers \& Operations Research, vol. 35, no. 7, pp. 2234-2247, 2008. 

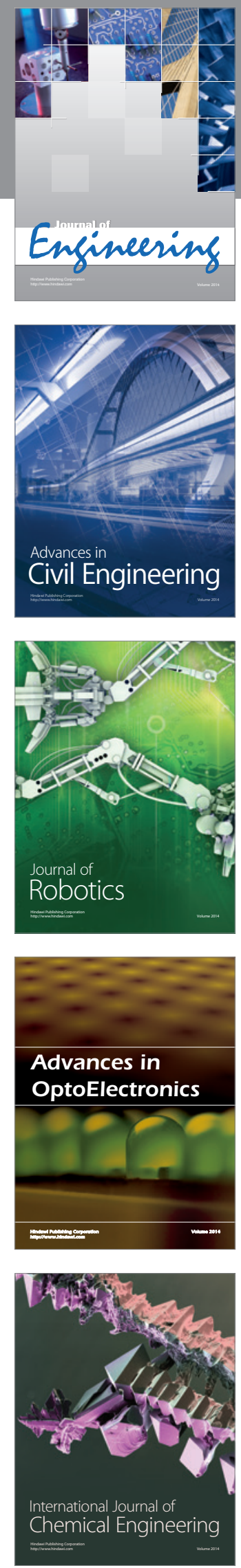

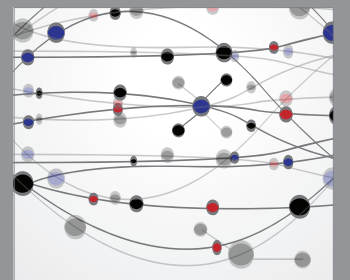

The Scientific World Journal
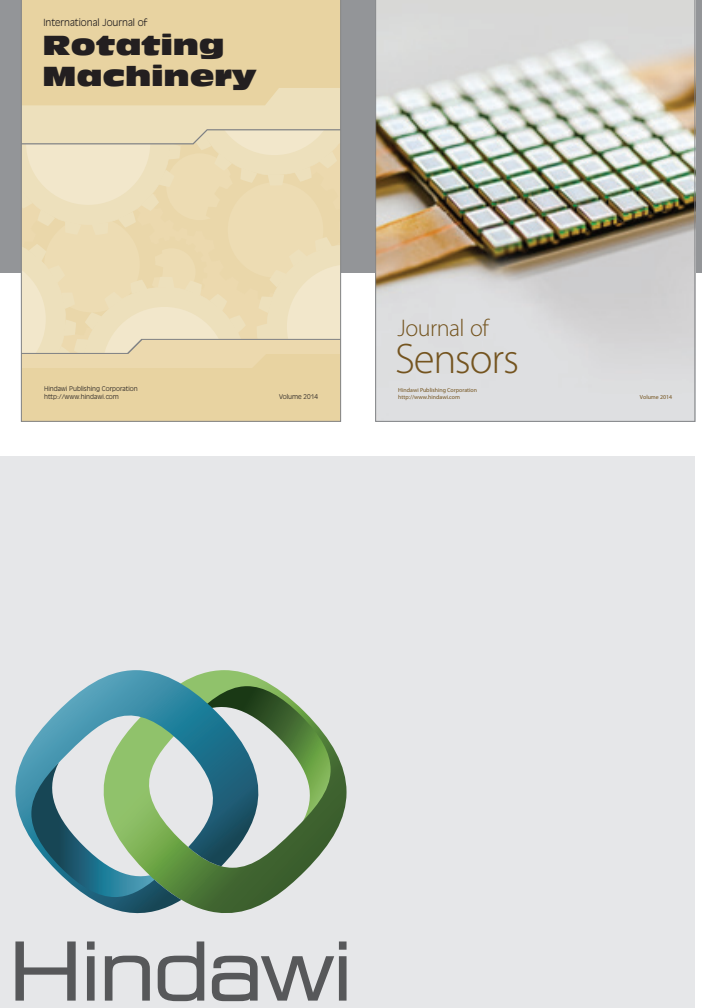

Submit your manuscripts at http://www.hindawi.com
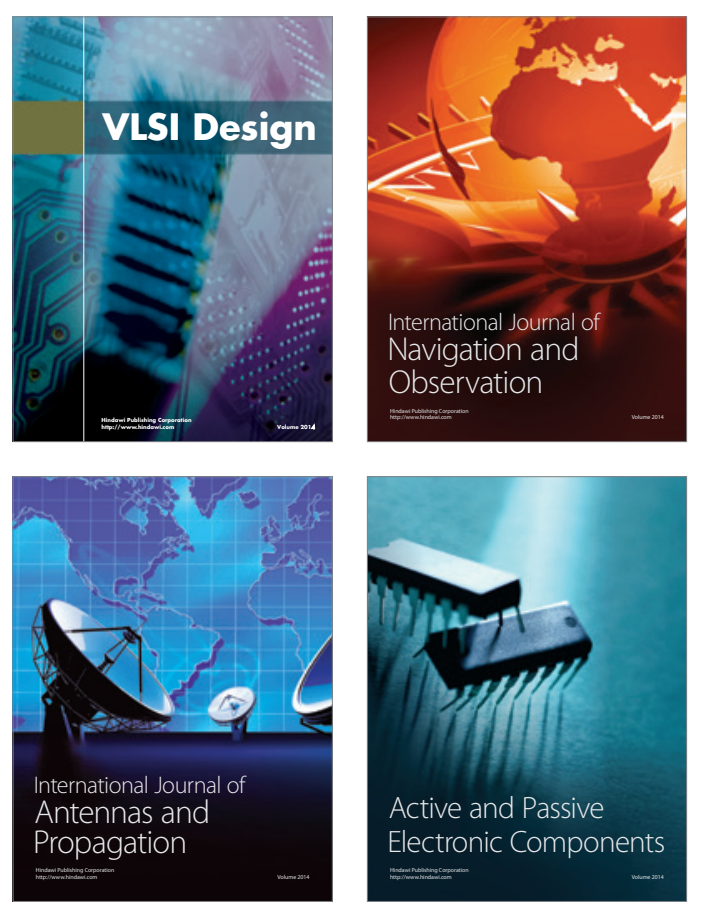
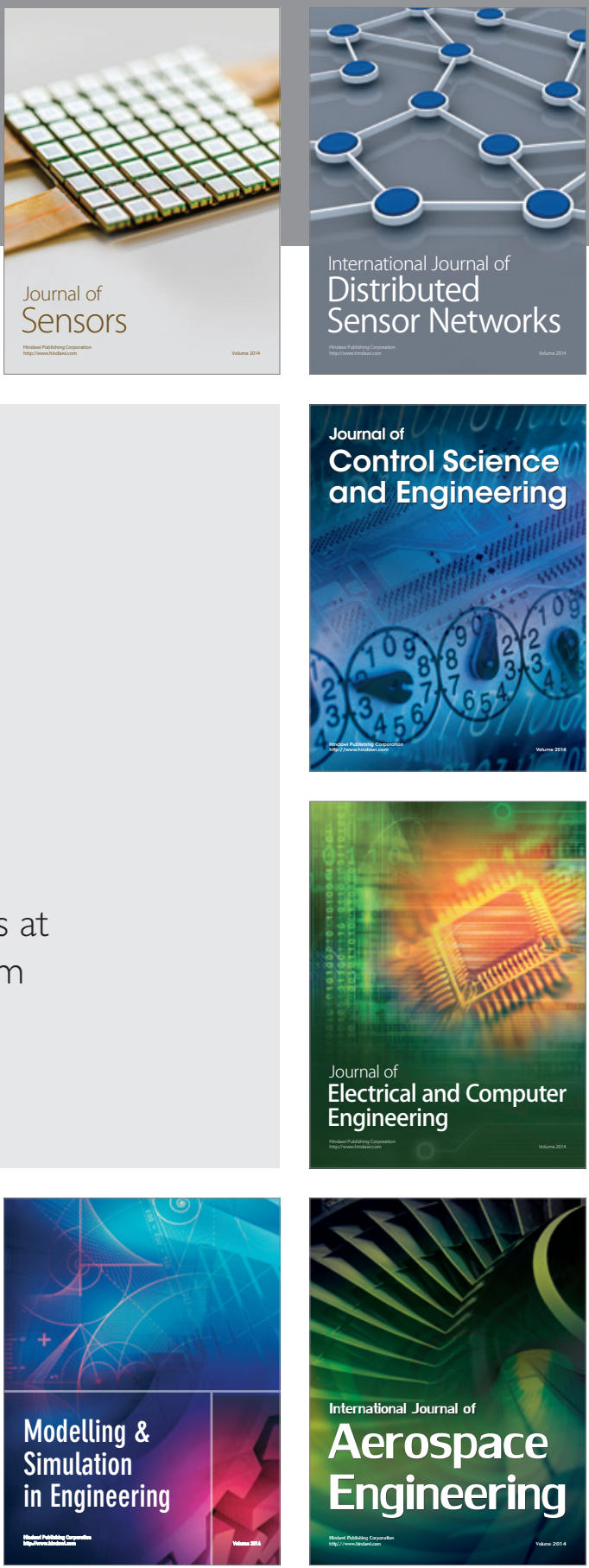

Journal of

Control Science

and Engineering
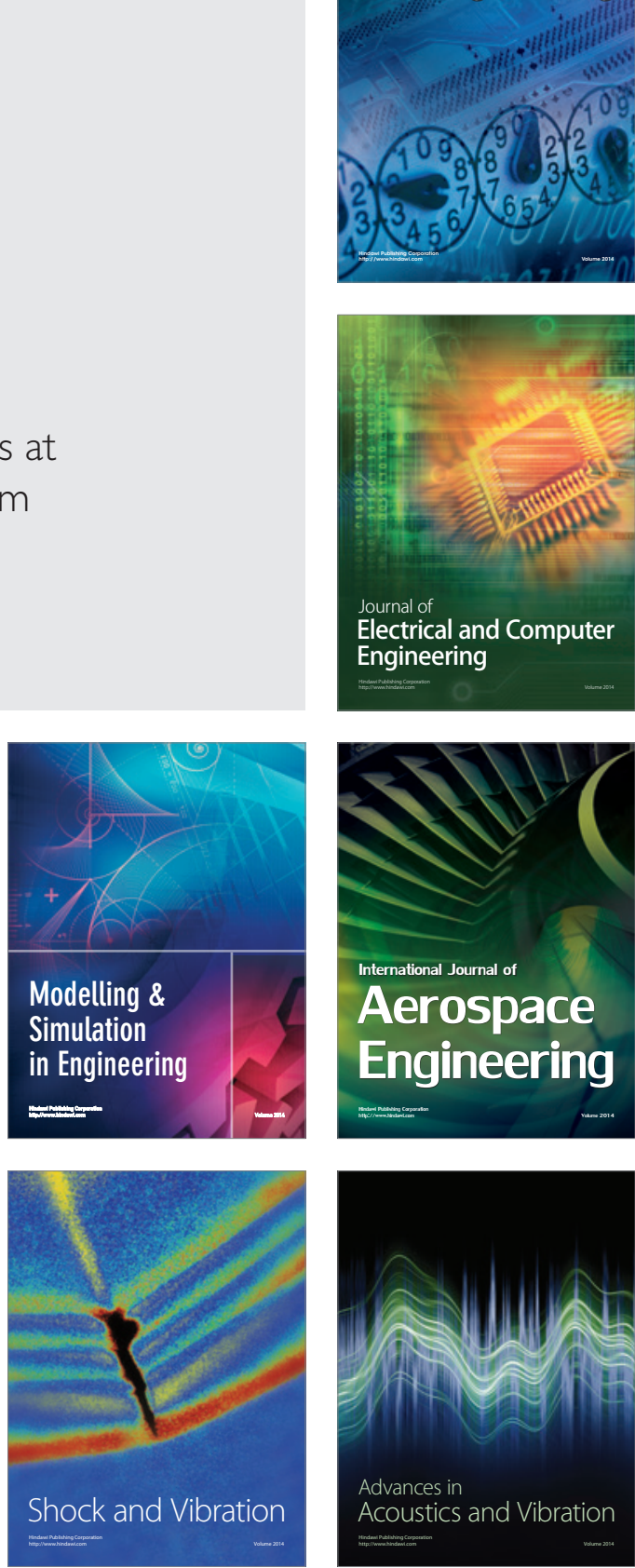